\title{
Problèmes aux limites non homogènes (VII)
}

\author{
par J, L. Lrons (Paris) et E. Magenes (Pavia)
}

Résumé - Voir l'introduction.

\section{Introduction.}

Soit $A$ un opérateur différentiel linéaire elliptique d'ordre $2 m$ dans un ouvert $\Omega$ de $R^{n}$, borné, de frontière $\Gamma$, et $B_{j}(j=0,1, \ldots, m-1)$ un système de $m$ opérateurs différentiels d'ordre $<2 m$ formant un système normal et recouvrant $A$ au sens de [2] et [20]. On cherche une "fonetion, $u\left({ }^{1}\right)$ solution de

$$
A u=f \text { dans } \mathrm{Q}, B_{j} u=g_{j} \text { sur } \mathrm{r}, j=0, \ldots, m-1
$$

les $f$ et $g_{j}$ étant donnés dans $\Omega$ et sur $\Gamma$.

Dans les articles (III) $\cdot(V)$ de [13] nous avons considéré ces problèmes pour les $f$ et $g_{j}$ dans diverses classes d'espaces construits à partir des espaces de Sobolev par la théorie de l'interpolation (l'article (II) se bornait au cas hilbertien).

Dans l'article $(V I)$ nous avons, en particulier, résolu le problème $\left(^{*}\right)$ pour les $g_{j}$ distributions quelconques sur $\Gamma$ (en supposant que $\Gamma$ est une variété indéfiniment différentiable). Alors $u$ et $A u$ étaient assujettis à des conditions de croissance an voisinage de $\Gamma$; grosso-modo, si $u$ et $A u$ ne croissent "pas trop vite, au voisinage de $\Gamma$ alors l'on peul définir les $B_{j} u$ sur $\Gamma$ comme distributions sur $\Gamma^{(2)}$.

Dans l'article présent, nous supposerons que $u$ est une distribulion qnelconque dans $\Omega$ et que Au est une distribution qui "ne croit pas trop vite, au voisinage de $\Gamma$ (condition précise: $A u \in \Xi^{\prime}(\Omega)$ défini au n. 5 ).

Alors, la frontière $\Gamma$ étant analytique et les coefficients de $A$ et $B_{i}$ étant analytiques, on peut définir les $B$, u sur $\Gamma$ : ce sont des fonctionnelles analytiques.

(1) Les hypothèses précises sont données ci après.

$\left.{ }^{2}\right)$ La condition nécessaire et suffisante portant sur Au pour que l'on puisse définir, par exemple, la trace de $u$ sur $\Gamma$ en tant que distribution sur $\Gamma$ ne semble pas connue. L'article (VI) donne des conditions suiffisantes. 
En particulier donc toute fonction qui est solution d'une équation $A u=0$, avec $A$ elliptique et à coefficients analytiques, admet une trace sur $\Gamma$ qui est une fonctionnelle analytique.

Nous montrons outre, que, $f$ étant donné dans $\mathbb{E}^{\prime}(\Omega)$ et les $g$, étant des fonctionnelles analytiques données, on peut résoudre le problème (*), avec, dans un certain sens, "le théorème de l'alternative,,

Ce travail a été inspiré par une intéressante conférence de G. Cimmino [4] (voir anssi sa communication au Congrès international de Stockolm, 1962) $\left({ }^{3}\right)$, dans laquelle il posait la question d'étudier les problèmes aux limites pour les opérateurs linéaires elliptiques en supposant que les données aux limites soient des fonctionnelles analytiques et il donnait des résultats sur ce problème dans des cas particuliers. Nous avons observé que certaines des méthodes que nous avons utilisées dans les articles précédents permettent de résoudre cette question de façon générale: on part des résultats "très réguliers", on les "transpose, et on interprète les résultats "transposés, à l'aide de théorèmes de traces. Par contre les détails techniques diffèrent essentiellement de ceux des articles précédents. Sonlignons aussi que l'on n' utilise ici ni la théorie de l'interpolation des opérateurs linéaires, ni la théorie des problèmes aux limites dans $L^{p}, p \neq 2$ : l'article présent pent donc être lu, pour l'essentiel, indépendamment des articles précédents.

Quelques problèmes, qui nẹ nous semblent pas dénnés d'intérêt et qui nous semblent résolubles avec nos méthodes, se posent dans ces directions: 1) cas où la frontière et les coefficients, au lieu d'être analytiques, sont dans des classes de Gevrey; 2) cas où $u$ n'est plus une distribution, mais une ultra-distribution (cf. [18], [19]) ou dans certaines classes de "fonctions généralisées," considérées par GeLFand-SoHilov [7].

Le plan de l'article est le suivant:

1. Espaces $\mathscr{H}(\Gamma), \mathscr{H}^{\prime}(\Gamma)$.

2. Opérateurs differentiels et problèmes aux limites considérés.

3. Nouveaux espaces fonctionnels.

4. Transposition.

5. Espaces $\Xi(\Omega), \Xi^{\prime}(\Omega)$.

6. Choix de la forme $L$.

7. Lemmes préliminaires pour la définition des traces.

8. Un théorème de traces.

9. Problèmes aux limites non homogènes.

10. Bibliographie.

11. Index des notations principales.

Mars 1963.

(3) On doit évidemment signaler aussí les travaux de Kötme [11] et Tuldmann [22] sur la trace des fonctions holomorphes. 
J. L. Lions - E. Magenes: Problèmes aux limites non homogènes (VII) 203

1. - EsPaCes $\mathfrak{H}(\Gamma)$ et $\mathfrak{H}^{\prime}(\Gamma)$.

1.1. - On considère dans $R^{n}$ un ouvert $\mathbf{\Omega}$ borné de frontière $\Gamma$; on suppose $q u \Theta \Gamma$ est une variété (compacte) analytique réelle de dimension $n-1$, orientée, $\mathbf{Q}$ étant d' un seul côté de $\left.\Gamma .{ }^{*}\right)$

Nous utiliserons constamment l'espace vectoriel $\mathscr{H}(\mathbf{\Gamma})$ des fonctions analytiques sur $\mathrm{\Gamma}$.

II est bien connu que l'on peat introduire dans $\mathcal{H}(\mathrm{C})$ une topologie d'espace vectoriel topologique localement convexe complet et plus précisément d'espace (£F) selon Grothendieck [8]. Habituellement on plonge par une procéde "standard, $\Gamma$ dans une variété analytique complexe $\tilde{\Gamma}$ de dimension $n-1$, de façon que si $f$ est une fonction de $\mathscr{H}(\Gamma)$, il existe un entourage fermé $U$ de $\Gamma$ dans $\tilde{\Gamma}$, dépendant de $f$, dans lequel on puisse prolonger $f$ en une fonction holomorphe dans l'intérieur de $U$ et continue dans $U$; on considère alors une suite $U_{k}(k=0,1,2, \ldots)$ décroissante d'entourages fermés de $\Gamma$ dans $\tilde{\Gamma}$ tels que $\bigcap_{k} U_{k}=\Gamma$ et les espaces $\mathscr{H}\left(U_{k}\right)$ des fonctions holomorphes dans l'intérieur de $U_{k}$ et continues dans $U_{k}$ avec la topologie habituelle de la convergence uniforme dans $U_{k}$; et enfin on définit la topologie dans $\mathscr{H}(\Gamma)$ comme la topologie limite inductive (of. par ex. Bourbaki [23! Köthe [12]) des topologies induites par l'application de $\mathscr{H}\left(U_{k}\right)$ dans $\mathscr{H}(\Gamma)$ obtenue par restriction des fonctions de $\mathcal{H}\left(U_{k}\right)$ sur $\Gamma$.

Il est, semble-t'il, préférable, dans notre cas, de définir la topologie dans $\mathcal{H}(\Gamma)$ en ne sortant pas de la variété $\Gamma$.

Or il est bien connu que les fonctions analytiques, par ex. dans une sphère de $R^{n-1}$, peuvent être caractérisées par des majorations des dérivées de tous les ordres (du type $\sup _{x}\left|\frac{\partial^{k} f(x)}{\partial x_{1}^{k 1} \ldots \partial x_{n-1}^{k}}\right| \leq k_{1} ! \ldots k_{n-1} ! L M^{k}$ ).

Tout récemment Kotake ef Narastmhan [9] ont donné une autre caractérisation en utilisant, au lieu des dérivées d'ordre $k$, les puissances $\Lambda^{k}$ d'un operateur $\Lambda$ lineaire elliptique quelconque à coefficients analytiques (cf. aussi NeLson [17]) et des majorations intégrales.

On peut done essayer d'etendre ces caractérisations à l'espace $\mathfrak{H}(\Gamma)$ et de les utiliser pour la definition de la topologie dans $\mathscr{H}(\Gamma)$. En fait pour l'utilisation de la première caractérisation on peut voir par ex. la confé rence [4] de Cimmino. Dans ce travail nous utiliserons les résultats de KOTAKE-NARASINHAM en prenant sur $\Gamma$ l'opérateur intrinsèque de LAPLACEBeltrami $\Delta$ et ses itérés $\Delta^{k}, k=1,2, \ldots$

(4) Ponr les notions sur les varietes différentiables, les fonctions et les opérateurs différentiels sur les varietés que nous utiliserons dans ce travail, on peut voir par ex. De Rham [5] et Aronszajn. Milgram [2]. 
Fixons d'abord quelques notations; on désignera par $C^{0}(\Gamma)\left(\right.$ resp. $\left.L^{2}(\Gamma)\right)$ l'espace des fonctions continues (resp. des classes des fonotions de carré sommables sur $\Gamma$ pour la mesure de surface $d \sigma$ ) muni de la norme

$$
\|f\| C_{0}(\Gamma)=\sup _{x \in \Gamma}|f(x)|
$$

(resp.

$$
\left.\|f\|_{L^{2}(\Gamma)}=\left(\int_{\Gamma}|f(x)|^{2} d \sigma\right)^{\frac{1}{2}}\right)
$$

Nous considérons $\Gamma$, ce qui est possible, comme une variété de Rremann en prenant sur $\Gamma$ la métrique riemanienne induite par la métrique de $R^{n}$.

On peat alors considérer l'opérateur de Laplace-Beltram $\Delta$ (cf. par ex. De Rham [5] pag. 125) qui applique chaque fonction indéfiniment différentiable sur $\Gamma$ dans une fonction indéfiniment différentiable sur $\Gamma$. On a alors la

Proposition 1.1: - L'espace $\mathfrak{H}(\Gamma)$ est l'ensemble des fonctions $f$ indéfi. niment différentiables sur $\mathrm{T}$ telles $q u^{\prime} i l$ existe dewx constantes $L_{2}$ et $M_{2}$ (dépendantes de $f$ ) telles que, pour tout nombre entier $k \geq 0$, on ait

$$
\left\|\Delta^{k} f\right\|_{L^{2}(\mathbf{\Gamma})} \leq L_{2}(2 k) ! M_{2}^{k}, \quad \quad\left(\Delta^{\prime \prime} f=f\right)
$$

Démonstration - En effet par définition des variétés analytiques réelles compactes, il existe un recouvrement ouvert de $\Gamma,\left\{\Gamma_{i}\right\}, i=1 \ldots, \nu$, et pour chaque $\Gamma_{i}$ un systéme de coordonnées locales, $x \rightarrow \Theta_{i}(x) \equiv\left\{x_{i, 1}(x), \ldots, x_{i, n-1}(x)\right\}$ c'est-à-dire un homéomorphisme $\Theta_{i}$ de $\Gamma_{i}$ sur une sphére $S^{n-1}$ ouverte de $R^{n-1}$, satisfaisant aux conditions de compatibilité usuelles analytiques. Alors il suffit de remarquer que, en exprimant sur chaque $\Gamma_{i}$ l'opérateur $\Delta$ à l'aide de coordonnées locales, on obtient dans $S^{n-1}$ un opératenr $\Lambda_{i}$ différentiel linéaire elliptique du deuxième ordre à coefficients analytiques dans $S^{n-1}$ et appliquer à cet opérateur les résultats de KotaKE-NARAsinham [9], theorem 1.

Une deuxième caractérisation de $\mathfrak{H}(\Gamma)$ est la suivante.

Propositiox 1.2: - L'espace $\mathfrak{H}(\Gamma)$ esl l'ensemble des fonctions $f$ indéf $i$ niment différentiables sur $\Gamma$ telles $q u$ il existe deux conslantes $L_{0}$ et $M_{0}$ (dépendantes de $f$ ) telles que pour tout entier $k \geq 0$, on ait

$$
\left\|\Delta^{k} f\right\|_{C^{0}(\mathbf{S})} \leq L_{0}(2 k) ! M_{0}^{k}, \quad\left(\Delta^{0} f=f\right)
$$


J. L. Lions - E. Magenes : Problèmes aux limites non homogènes (VII) 205

Démonstration - En utilisant la Prop. 1.1, il suffit de montrer que: si $f$ est une fonction indéfiniment différentiable sur $\Gamma$ telle que, pour $k=0,1,2, \ldots,\left(\begin{array}{ll}1 & 1\end{array}\right)$ soit vraie pour $L_{2}$ et $M_{2}$ constantes convenables, alors (1.2) est vraie pour $L_{\lrcorner}$et $M_{0}$ constantes convenables, et réciproquement.

Or (1.2) entraine évidemment (1.1), puisque

$$
\|f\|_{L^{2}(\Gamma)} \leq c\|f\|_{c^{o}(\Gamma)}, \quad c \text { constante. }
$$

Pour démontrer que (1.1) entraine (1.2), utilisons encore le recouvrement ouvert de $\Gamma,\left\{\Gamma_{i}\right\}, i=1, \ldots, \vee$, et le système de coordonnées locales $\left\{\Theta_{i}\right\}$, introduits dans la démonstration de la Prop. 1.1.

Il existe certainement pour chaque $\Gamma_{i}$ un compact $k_{i}$ de $\Gamma$ tel que $k_{i}$ soit contenu dans $\Gamma_{i}$ et que $\bigcup_{i=1}^{\nu} k_{t}=\Gamma$. Soit $f$ une fonction indéfiniment différentiable sur $\Gamma$ et soit $f_{i}$ "l'image, dans $S^{n-1}$ de $f$ (restreinte à $\Gamma_{i}$ ) par utilisation de l'homéonorphisme $\Theta_{i}$; alors $\Delta^{k} f$ a pour image $\Lambda_{i}^{k} f_{i}$, $k=1,2, \ldots$ et $k_{i}$ a pour image un compact $\tilde{k}_{i} \subset S^{n-1}$. Mais les résultats désormais classiques sur la "regularisation à l'intérieur, des équations elliptiques nous donnent que, si $\varphi \in L^{2}\left(S^{n-1}\right)$, avec $\Lambda_{i}^{\mu} \rho \in L^{2}\left(S^{n-1}\right), \mu$ entier $\geq 1$. alors $\varphi \in H^{2 \mu}\left(S^{*}\right)$ pour chaque sphère $S^{*}$ intérieure à $S^{n-1}\left({ }^{5}\right)$ et

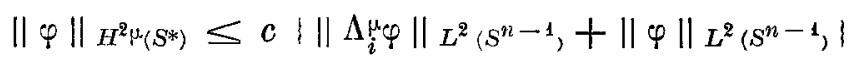

d'où, par utilisation des inégalités de SoBoLEv, on déduit pour $\mu$ assez grand $\left(\frac{1}{2}-\frac{2 \mu}{n-1}<0\right)$, que $\varphi$ est continue sur $S^{*}$ et que

$$
\sup _{y \in S^{*}}|\varphi(y)| \leq C^{*}\left(\left\|\Lambda_{i}^{\mu} \varphi\right\|_{L^{2}\left(S^{n-1}\right)+\|\varphi\| L^{2}\left(S^{n-1}\right)}\right)
$$

Donc en conclusion on peut dire qu'il existe des constantes $c_{i}$, $i=1, \ldots, \nu$, telles que

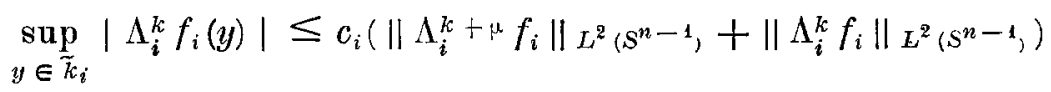

pour tout $k$ entier $\geq 0, \mu$ étant fixé assez grand.

(5) $H^{2} \mu\left(S^{*}\right)$ est l'espace des fonctions de carré sommable dans $S^{*}$ avec toutes ses dérivées d'ordre $\leq 2 \mu$. 
Alors on a aussi

$$
\left\|\Delta^{k} f\right\| c_{0}(\mathbf{\Gamma}\rangle \leq c\left(\left\|\Delta^{k+1} f\right\| L_{L^{2}(\mathbf{\Gamma})}+\left\|\Delta^{k} f\right\| L_{L^{2}(\Gamma)}\right)
$$

d'où, en utilisant (1.1)

$$
\left\|\Delta^{k} f\right\| c^{0}(\Gamma) \leq c L_{2} M_{2}^{k}(2 k) !\left[1+M_{2}^{\mu}(2 k+1) \ldots(2 k+2 \mu)\right]
$$

Mais $(2 k+1) \ldots(2 k+2 \mu) \leq c^{\prime} \mathrm{e}^{2 k_{\mu}}$, done

$$
\left[1+M_{2}^{\mu}(2 k+1) \ldots(2 k+2 \mu)\right] \leq c^{\prime \prime} M^{k}\left(c^{\prime}, c^{\prime \prime}, M \text { constantes }\right)
$$

et alors on obtient (1.2) avec $L_{0}$ et $M_{0}$ constantes convenables.

Remarque 1.1. - On peut encore caractériser $\mathscr{H}(\Gamma)$, de façon analogue, comme l'espace des fonctions indéfiniment différentiables sur $\mathbf{I}$ telles qu'il existe deux constantes $L_{p}$ et $M_{p}$ (dépendant de $f$ ) telles' que pout tout entier $k \geq 0$, on ait

$$
\left\|\Delta^{k} f\right\| L^{n}(\mathrm{\Gamma}) \leq L_{p}(2 k) ! M_{p}^{k}
$$

avec $p$ fixé quelconque $1<p<+\infty$.

Cette remarque s'étend aux points 1.2 et 1.3 ei après; nous n'insisterons pas sur cela.

\subsection{Topologie sUR $\mathfrak{H}(\Gamma)$.}

Désignons par $\mathfrak{H}_{M, 0}(\Gamma)$ ( resp. $\mathscr{H}_{M, 2}(\Gamma)$ ) l'espace des fonctions $f$ de $\mathscr{H}(\Gamma)$ telles que l'on ait

$$
\left\|\Delta^{k} f\right\|_{C^{o}(\Gamma)} \leq L_{0}(2 k) ! M^{k} \quad k=0,1, \ldots
$$

avec $M$ fixé et $L_{0}$ dépendant de $f$ (resp.

$$
\left\|\Delta^{k} f\right\|_{L^{2}(\mathrm{~T})} \leq L_{2}(2 k) ! M^{k} \quad k=0,1, \ldots
$$

avec $M$ fixé, $L_{2}$ dépendant de f) 
On muni $\mathscr{H}_{M T, 0}(\Gamma)$ (resp. $\mathscr{H}_{M,-2}(\Gamma)$ ) de la norme

$$
\|f\|_{\mathcal{H}_{M, 0}(\boldsymbol{\Gamma})}=\sup _{k} \frac{1}{(2 k) ! M^{k}}\left\|\Delta^{k} f\right\| C_{(\Gamma)}
$$

(resp.

$$
\|f\|_{H_{M, 2}(\mathrm{\Gamma})}=\sup _{k} \frac{1}{(2 k) ! M^{k}}\left\|\cdot \Delta^{k} f\right\|_{L^{2}(\Gamma)}
$$

Pour cette norme $\mathfrak{H}_{M, 0}(\Gamma)$ (resp. $\mathcal{H}_{M, 2}(\Gamma)$ ) est un espace de BANACH.

On a, si $M<M^{\prime}$

$$
\mathscr{H}_{M, 0}(\Gamma) \subset \mathfrak{H}_{M^{\prime}, 0}(\Gamma), \quad \mathfrak{H}_{M, 2}(\Gamma) \subset \mathfrak{H}_{M^{\prime}, 2}(\Gamma)
$$

avec injection continue.

On munit alors $\mathscr{H} \Gamma$ ) de la topologie limite inductive des $\mathscr{H}_{M, 0}(\Gamma)$ (resp. $\mathfrak{H}_{M, 2}(\Gamma)$ ) pour $M=1,2, \ldots$ (ef. par ex. Bourbaki [32] Köthe [12]). Les topo-

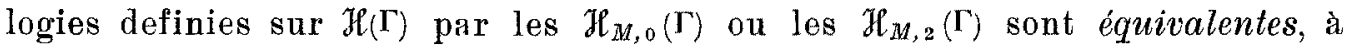
cause de ce $q u^{\prime}$ on a vu à propos des Propositions 1.1 et 1.2.

$L$ 'espace $\mathcal{H}(\Gamma)$ rentre ainsi dans la famille des espaces (SFF) au sens de Grothendieck [8] (consulter également Roumieu [18]. et Köthe [10] [11] [12]).

1.3. Espace $\mathfrak{H}^{\prime}(\Gamma)$.

On désigne par $\mathfrak{H}^{\prime}(\Gamma)$ l'espace dual (fort) de $\mathfrak{H}(\Gamma)$ (c'est un espace de fonctionnelles analytiques).

Nous allons démontrer la

Proposition 1.3: - Toute forme linéaire continue $u \rightarrow \mathcal{L}(u)$ sur $\mathfrak{H}(\Gamma)$ peut se représenter sous l'une des formes suivantes:

$$
\mathcal{L}(u)=\sum_{k=0}^{\infty} \int_{\Gamma} \Delta^{k} u d \mu_{k}
$$

avec $\mu_{k} \in\left(C^{n}(\Gamma)\right)^{\prime}$, mesure sur $\Gamma$, et $\sum_{k=0}^{\infty} M^{k}(2 k) !\left|\mu_{k}\right|<+\propto$ quel que soit $M>0\left(\mid \mu_{k} !=\right.$ variation tolale de $\left.\mu_{k}\right)$, el

$$
S(u)=\stackrel{\infty}{\Sigma}=0_{\Gamma}^{\infty} g_{k} \Delta^{k} u d \sigma
$$

avec $g_{k} \in L^{2}(\Gamma)$ et $\sum_{k=1}^{\infty} M^{k}(2 k) !\left\|g_{k}\right\| L^{2}(\mathrm{\Gamma}\rangle<+\infty$ quel que soit $M>0$. 
Démonstration. - Cette proposition résulte aussitôt des théorèmes de structure des formes linéaires continues sur les espaces considérés par KöTHE et Roumied. En fait par ex. pour démontrer (1.8) considérons l'espace $E$ des suites $F \equiv\left\{f_{n}, f_{1}, \ldots\right\}$ des fonctions $f_{k} \in L^{2}(\Gamma)$ telles qu'il existe des constantes $L$ et $M$, dépendant de la suite $F$, avec

$$
\left\|f_{k}\right\|_{L^{2}(\Gamma)} \leq L(2 k) ! M^{k} \quad k=0,1, \ldots
$$

On désigne par $E_{M}$ le sous espace de $E$ formé des sujtes telles que (1.9) ait lieu, avec $M$ fixé, $L$ pouvant dépendre de la suite; on munit $E_{M}$ de la norme

$$
\|F\|_{E_{M}}=\sup _{k} \frac{1}{(2 k) ! M^{k}}\left\|f_{k}\right\| L_{L^{2}(\mathrm{\Gamma})} \quad F=\left\{f_{k} \mid\right.
$$

et enfin on munit $E$ de la topologie limite inductive des topologies de $E_{M}$, pour $M=1,2 \ldots$

Alors $E$ est un (2F) selon [8] et $\mathscr{H}(\Gamma)$ peut être considéré comme un sous-espace vectoriel fermé de $E$, en posant, pour tout $f \in \mathscr{H}(\Gamma), F=\left\{\Delta^{k} f\right\}$.

Mais on connait (cf. par ex. Roumieu [18], Prop. 3 pag. 45) l'expression des formes linéaires continues sur $E, F \rightarrow \mathfrak{L}(F)$ :

$$
\mathcal{S}(F)=\sum_{k=1}^{\infty} \int_{\Gamma} g_{k} f_{k} d \sigma
$$

où les $g_{k} \in L^{2}(\Gamma)$ et $\sum_{k=1}^{\infty} M^{k}(2 k) !\left\|g_{k}\right\|_{L^{2}(\Gamma)}<+\infty$ quel que soit $M>0$. Toute forme linéaire continue sur $\mathscr{H}(\Gamma)$ pouvant, d'après le théorème de HaHN-Banach, se prolonger en une forme linéaire continue sur $E$, on a le résultat.

On démontre (1.7) de façon analogue.

Remarque 1.2. - Signalons que les $\mu_{k}$ et $g_{k}$ ne sont pas définis de façon unique.

\section{Opérateurs différentiels et problèmes aux limites considérés.}

2.1. Dans l'ouvert $\Omega$ on considère un opérateur différentiel $A$ donné par

$$
A u=\underset{|l|,|h| \leq m}{\Sigma}(-1)^{|l|} D^{l}\left(a_{l h}(x) D_{h} u\right)
$$

on utilise les notations habituelles; par ex.: $h=\left(h_{1}, \ldots, h_{n}\right), h_{i}$ entier $\geq 0$,

$$
|h|=h_{1}+\ldots+h_{n}, D^{n}=\frac{\partial|h|}{\partial x_{1}{ }^{{ }_{1}} \ldots \partial x_{n}{ }^{h_{n}}} .
$$


J. L. Lrons - E. Maggaes: Problimes aux limites non homogènes (VII) 209

On fera sur $A$ les hypothèses suivantes:

(B) $A$ est proprement elliptique dans $\bar{\Omega}(\bar{\Omega}=\mathbf{Q} \cup \Gamma)$, (terminologie de [20]) (6) (QQ1) les cofficients $a_{l h}$ sont analytiques dans $\overline{\mathrm{\Omega}}$.

On considère dans $\mathbf{Q}$ les problèmes aux limites suivants (où, naturellement, les hypothèses de différentiabilité seront précisées par la suite):

$$
\left\{\begin{array}{l}
A u=f \text { dans } \Omega, \quad f \text { donné } \\
B, u=g_{j}, j=0,1, \ldots, m-1 \text { sur } \Gamma
\end{array}\right.
$$

où les $g_{j}$ sont données sur $\Gamma$, et où

$$
B_{j} u=\underset{|h| \leq m_{j}}{\Sigma} b_{j h}(x) D^{h} u, \quad 0 \leq m_{j}<2 m ;
$$

On suppose que

(Q2) les coefficients $a_{j h}$ sont analytiques sur $\Gamma$;

et que

(ソ) le système des $B_{j}$ est normal au sens de Aronszajn-Milgram [2] ( ${ }^{7}$ );

(R) le système des $B_{j}$ recouvre $A$ au sens de AGMON-DoUgLIS-NIRENGERG [1] et de Schechter $[20]\left({ }^{8}\right)$.

On désigne par $A^{*}$ l'adjoint (formel) de $A$, donné par

$$
A^{*} u=\sum_{|u|,|h| \leq m}(-1)|u| D^{l}\left(\bar{a}_{h l}(x) D^{n} u\right)
$$

On va introduire un adjoint formel du problème (2.2).

(6) C'est à dire pour tout $x \in \bar{\Omega}$ et tout couple de vecteurs réels $\xi$ et $\xi$ linéairement independants, le polynome en $\tau \sum_{|l|,|h|=m} a_{l h}(x)\left(\xi+\tau \xi^{\prime}\right)^{l}+h$ possède $m$ racines de partie imaginaire positive.

(7) Cest à dire tel que $m_{j} \neq m_{i}$ pour $j \neq i$ et que pour tout $x \in \Gamma$ et tout vecteur $\xi \neq 0$ et normal à $I$ en $x$ on ait $\underset{|h|=m_{j}}{\Sigma} b_{j h}(x) \xi^{h} \neq 0$.

(8) C'est à dire tel que pour tout $x \in \Gamma$ les polynomes on $\tau, \underset{|h|=m_{j}}{\Sigma} b_{j h}(x)\left(\xi^{\prime}+\tau \xi\right)^{h}$, $j=0, \ldots, m-1$, où $\xi^{\prime}$ est un vecteur quelconque réel $\neq 0$ et tangent à $\Gamma$ en $x$, et $\xi$ un vecteur quelconque réel $\neq 0$ et no:mal à $\Gamma$ en $x$, sont linéairement indépendants "modulo, le polynome ${ }_{i=1}^{m}\left(\tau-\tau_{i}(\xi\right.$, $\left.)\right)$ où $\tau_{i}(\xi$,,$\xi)$ sont les racines de partie imaginaire positive du polynome $\underset{|l|, h \mid=m}{\Sigma} a_{l h}(x)\left(\xi^{\prime}+\tau \xi\right)^{l+h}$. 
Notons d'abord que l'on peut toujours - et de façon non unique - compléter le système $\left.\mid B_{j}\right\}_{j=0}^{m-1}$ par un système $\left\{O_{j}^{j=0} j_{j=0}^{m-1}\right.$, où les $O_{j}$ sont des opérateurs differentiels d'ordre $\mu_{j} \leq 2 m-1$ à coefficients analytiques sur $\Gamma$, tels que le système $\left\{B_{0}, \ldots, B_{m-1}, C_{0}, \ldots, C_{m-1}\right\}$ soit normal et de DrRichlate (i. e. les ordres $m_{j}, \mu_{s}, j=0, \ldots \ldots, m-1$, parcourent exactement l'ensemble $0,1, \ldots, 2 m-1$ ).

Alors (cf. [2], [20]) il existe $2 m$ opérateurs $B_{j}^{*}, O_{j}^{*}, j=0, \ldots \ldots, m-1$, à coefficients analytiques sur $\Gamma$, avec

$$
\begin{aligned}
& \text { ordre } B_{j}^{*}=2 m-\mu_{j}-1, \\
& \text { ordre } C_{j}^{*}=2 m-m_{j}-1,
\end{aligned}
$$

le système $\left\{B_{0}^{*}, \ldots, B_{m-1}^{*}, C_{0}^{*}, \ldots, C_{m-1}^{*}\right\}$ étant normal et de DirichLet et tels que l'on ait la formule de Green:

$$
(A u, v)-\left(u, A^{*} v\right)=\sum_{j=0}^{m-1} \int_{\Gamma}\left(C_{j} u\right)\left(\overline{B_{j}^{*} v}\right) d o-\sum_{j=0}^{m-1} \int_{\Gamma}\left(B_{j} u\right)\left(\overline{C_{j}^{*} v}\right) d \sigma
$$

puor $u, v$ suffisamment différentiables, et où $(f, g)=\int_{a} f \bar{g} d x$.

Ceci posé, le problème

$$
\left\{\begin{array}{l}
A^{*} v=\varphi \\
B_{j}^{*} v=\psi_{1}, \quad j=0, \ldots, m-1
\end{array}\right.
$$

sera dit problème adjoint (relativement à la formule de GreEN (2.5)) du problème (2.4).

2.2. Sous les hypothèses précédentes l'opérateur $A^{*}$ définit, après passage au quotient convenable (cf. détails dans la suite), un isomorphisme d'un certain espace $\Phi$ de classes de fonctions (satisfaisant à $B_{j}^{*} u=0$ sur $\Gamma$, si $u \in \Phi)$ sur un espace fonctionnel $\Psi$.

Nous désirons ici prendre $\Psi$ le "plus petit,, possible. Nous prendrons alors $\Psi=\mathfrak{D}(\Omega)$, espace des fonctions indéfiniment différentiables et à support compact dans $\mathbf{Q}$ muni de la topologie habituelle de $L$. Schwartz [21] (et en fait un sous espace fermé de $\mathfrak{D}(\boldsymbol{\Omega})$, parce que $\Phi$ est oblenu comme quotient). Les hypothèses $(\mathfrak{Q} 1)$ et $(\mathfrak{Q} 2)$ interviendront essentiellement pour l'interprétation des résultats, après transposition de l'isomorphisme $A^{*}$ de $\Phi$ sur $\Psi$, 
Remarque 2.1. - On pourrait prendre pour $\Psi$ un espace encore plus petit: un sous espace fermé des espaces $\mathscr{D}\left(\Omega ; M_{(p)}\right)$ tels qu'ils sont étudiés dans [19]. Nous ne poursuivrons pas ce point de vue ici.

\section{Noureaux espaces fonctionnels.}

3.1. On désigne par $\mathscr{F}_{A^{k}, B^{*}}$ l'espace des fonctions $u \in \mathfrak{D}(\overline{\mathbf{\Omega}})$ telles que $A^{*} u \in \mathfrak{D}(\Omega)$ et $B_{j}^{*} u=0$ sur $\Gamma, j=0,1, \ldots, m-1$.

Ici $\mathfrak{D}(\bar{\Omega})$ désigne d'espace des fonctions indéfiniment différentiables sur $\overline{\mathbf{Q}}$, muni de la topologie naturelle d'espace de FrÉcher.

On-munit $\mathscr{F}_{A^{*}, B^{*}}$ de la topologie de limite inductive ([6]) des espaces $\mathfrak{F}_{\boldsymbol{A}^{*}, B^{*}}^{x}=\left\{u \mid u \in \mathscr{D}(\overline{\mathbf{\Omega}}), A^{*} u \in \mathscr{D}_{K \chi}, B_{j}^{*} u=0, j=0, \ldots, m-1\right\}$, ôิ $K_{\alpha}=$ suite croissante de compacts $\subset \Omega$, de réunion $\Omega$, chaque $\mathscr{F}_{A^{*}, B^{*}}^{x}$ étant muni de la topologie naturelle d'éspace de Frechet.

On définit de même $\mathscr{F}_{A, B}$.

On désigne par $N$ (resp. $\left.N^{*}\right) l^{\prime}$ espace des $u \in \mathscr{F}_{A}, B$ (resp. $\mathscr{F}_{A^{*}, B^{*}}$ ) tels que $A u=0$ (resp. $A^{*} u=0$ ).

Les espaces $N$ et $N^{*}$ sont de dimension finie ([3] [20]).

3.2. Grace á [1], [3], [20] et $N$ étant de dimension finie, on voit que,

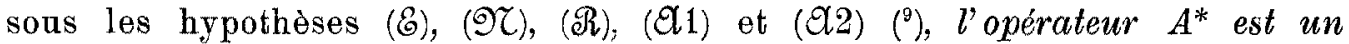
isomorphisme de l'espace quotient $\mathscr{F}_{A^{*}, B^{*} / N^{*}}$ sur l'espace $\mathfrak{D}(\Omega) ; N \mid$, où $\{\mathfrak{D}(\Omega) ; N \mid$ désigne le sous espace fermé de $\mathscr{D}(\boldsymbol{\Omega})$ des $v \in \mathfrak{D}(\boldsymbol{\Omega})$ telles que

$$
(v, w)=0 \text { pour tout } w \in N
$$

\section{Transposition.}

4.1. On transpose maintenant l'isomorphisme $A^{*}$ obtenu en 3.2. Il vient:

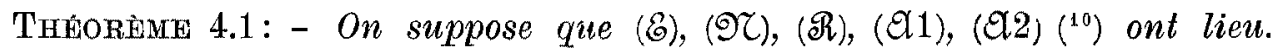
Soit $v \rightarrow L\left(v^{*}\right)$ une forme anti-linéaire continue sur $\mathfrak{F}_{A^{*} E^{*} / N^{*}} .1 l$ existe un élément $u$ et un seul de l' espace $\mid \mathfrak{D}(\Omega) ; N\}^{\prime}$ (dual de $\left.\{\mathscr{D}(\Omega) ; N\}\right)$ tel que

$$
<u^{*}, A^{*} v^{*}>=L\left(v^{*}\right) \quad \text { pour tout } v^{*} \in \mathscr{F}_{A^{*}, B^{*} / N^{*}}
$$

$u$ dépend continûnent de $L$.

$\left(^{9}\right)$ Signalons qu'il suffit que les coefficients $a_{h h}$ et $b_{j h}$ soient indéfiniment différentiables respectivement sur $\bar{\Omega}$ et $\Gamma$.

(10) of. note $\left({ }^{9}\right)$. 
Dans (4.1), le crochet désigne la dualité entre $\{\mathscr{D}(\Omega) ; N\}^{\prime}$ et $\{\mathfrak{D}(\mathbf{\Omega}) ; N\}$. Notons que $\left({ }^{11}\right)$

$$
\{\mathfrak{D}(\mathbf{\Omega}) ; N\}^{\prime}=\mathscr{D}^{\prime}(\mathbf{\Omega}) / N
$$

$\left(\mathfrak{D}^{\prime}(\boldsymbol{\Omega})=\right.$ dual de $\mathscr{D}(\boldsymbol{\Omega})$, espace des distribution sur $\left.\boldsymbol{\Omega}\right)$.

4.2. Le problème est maintenant: a) de choisir $L$ dans (4.1); b) d'interpreter (4.1), une fois $L$ choisi.

C'est dans l'interprétation de (4.1) que seront essentiallement atilisées les hypothèses d'analyticité des coefficients (cf. note $\left({ }^{9}\right)$ et $\left({ }^{10}\right)$ ).

Pour le choix de $L$, il convient d'introduire d'abord de nouveaux espaces fonctionnels sur $\mathbf{Q}: \Xi(\mathbf{Q}), \boldsymbol{\Xi}^{\prime}(\mathbf{\Omega})$; e'est l'objet du $N^{0}$ suivant.

\section{Espaces $\boldsymbol{\Xi}(\boldsymbol{\Omega}), \boldsymbol{\Xi}^{\prime}(\boldsymbol{\Omega})$.}

5.1. Espace $\Xi(\Omega)-$. On désigne par $\rho$ une fonction continue dans $\bar{\Omega}$, telle que $\rho(x)=d(x, \Gamma)$ (distance de $x$ à $\Gamma$ ) si $d(x, \Gamma) \leq \rho_{0}, \rho_{0}$ assez petit, et $\rho(x)=\rho_{0}$ si $d(x, \Gamma)>\rho_{0}$.

Ceci posé, on désigne par $\mathbb{\Xi}(\mathbf{\Omega})$ l'espace deś fonctions u telles que

$$
\rho^{|\alpha|} D^{x} u \in L^{z}(\Omega) \text { pour tout } \alpha=\left(\alpha_{1}, \ldots, \alpha_{11}\right)
$$

$\alpha_{i}$ entier $\geq 0$.

Cet espace est formé de fonctions indéfiniment différentiables dans $\bar{\Omega}$; c'est un espace de Frechet pour la famille de normes

$$
\left\|\rho^{|\alpha|} D^{\alpha} u\right\| L^{2}(\Omega)
$$

Naturellement:

$$
\mathfrak{D}(\bar{\Omega}) \subset \mathbb{\Xi}(\Omega) \text {, injection continue. }
$$

Vérifions le

Lemme $5.1-$ L'espace $\mathfrak{D}(\mathbf{\Omega})$ est dense dans $\mathbb{\Xi}(\mathbf{Q})$.

(11) En effet pour toute partie non vide $P$ d'un espace localement convexe separé $E$ l'ensemble bipolaire $P^{00}$ est identique au plus petit ensemble convexe cercle é fermé pour la topologie faible, qui contient $P$ (cf. par ex. [6] Prop. 1 pag. 64.) 
Démonstration - Soit $\delta_{\varepsilon}$ ane suite de fonetions de $\mathscr{D}(\Omega), \varepsilon \rightarrow 0$, telles que

$$
\begin{array}{ll}
\delta_{\varepsilon}(x)=1 \quad \text { si } \quad & d(x, \Gamma) \geq 2 \varepsilon, \\
\delta_{\varepsilon}(x)=0 & \text { si } \quad d(x, \Gamma) \leq \varepsilon,
\end{array}
$$

$\left|d(x, \Gamma)^{|\alpha|} D^{\alpha} \delta_{\varepsilon}(x)\right| \leq c^{\alpha}$ (constante dépendant de $\alpha$ ), pour tout $\alpha$.

Une telle suite existe.

Soit maintenant $u \in \mathbb{E}(\boldsymbol{\Omega})$; alors $\delta_{\varepsilon} u \in \mathfrak{D}(\mathbf{\Omega})$ et le lemme sera démontré si l'on vérifie que

$$
\delta_{\varepsilon} u \rightarrow u \text { dans } \Xi(\Omega) \text { lorsque } \varepsilon \rightarrow 0 .
$$

Pour cela, il faut montrer que $\rho^{|x|} D^{x}\left(\delta_{\varepsilon} u\right) \rightarrow \rho^{|x|} D^{x} u$ dans $L^{2}(\mathbf{Q})$; or $\delta^{\varepsilon} \rho^{|x|} D^{x} u \rightarrow \rho^{|x|} D^{x} u$ dans $L^{2}(\mathbf{Q})$; il suffit donc de montrer que

$$
\rho^{\mid \alpha}\left(D^{\beta} \delta_{\varepsilon}\right)(D r u) \rightarrow 0 \text { dans } L^{2}(\boldsymbol{\Omega}),|\beta| \geq 1,|\beta|+|\gamma|=|\alpha| \text {, lorsque } \varepsilon \rightarrow 0 \text {. }
$$

On $p^{|\alpha|}\left(D^{\beta} \delta_{s}\right)(D r u)$ est nulle si $d(x, \Gamma) \geq 2 \varepsilon$ (car $\left.|\beta| \geq 1\right)$, de sorte que, d'après le théorème de LeBESGUE, on a (5.3) en notant que

$$
\left|\rho^{\mid \boldsymbol{\alpha}}\left(D^{\beta} \delta_{\varepsilon}\right)(D \curlyvee u)\right|=\mid\left[\rho^{\prime \beta \mid}\left(D^{\beta} \delta_{\xi}\right)\right]\left[\rho^{\mid \gamma} D r u\left|\leq c_{\beta}\right| \rho^{|\gamma|} D^{\curlyvee} u \mid \in L^{2}(\Omega) .\right.
$$

Le lemme est démontré.

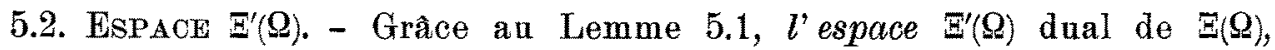
s'indentifie à un sous espace de $\mathscr{D}^{\prime}(\boldsymbol{\Omega})$. Toute forme linéaire $u \rightarrow M(u)$ continue sur $\Xi(\Omega)$ peut s'écrire:

$$
M(u)=\sum_{\text {fini } \Theta} \int_{\Omega} g_{\alpha} \rho^{|\alpha|} D^{\alpha} u d x, \quad g_{\alpha} \in L^{2}(\mathbf{\Omega}),
$$

d'où la

Propostrion 5.1. - L'espace $\Xi^{\prime}(\mathbf{\Omega})$ est formé des distributions $f$ pouvant se représenter (de façon non unique) sous la forme

$$
f=\sum_{f i n i \theta} D^{\alpha}\left(p^{|\alpha|} f_{\alpha}\right), \quad \quad f_{\alpha} \in L^{2}(\mathbf{\Omega})
$$

(on a pose : $\left.f_{\alpha}=(-1)^{|\boldsymbol{\alpha}|} g_{\alpha}\right)$. 
On peut alors démontrer ausssi la

Proposition 5.2. - L'espace $\Xi(\mathbf{Q})$ est réflexif.

Démonstration - En fait il suffit de montrer que ehaque partie bornée ef faiblement fermée de $\Xi(\mathbf{\Omega})$ est faiblement compacte (of. [6] pag. 79); or si $\left\{u_{i}\right\}$ est une suite bornée dans $\Xi(\Omega)$ il existe $M_{\alpha}$ tel que

$$
\left\|\rho^{|\alpha|} u_{i}\right\|_{L^{2}(\Omega)} \leq M_{\alpha} \quad \text { pour tout } \mathrm{i}
$$

Alors en utilisant le procédé «diagonal» on peut extraire de $\left\{u_{i}\right\}$ une sous-suite $\left\{u_{y}\right\}$ telle que $\rho^{|\alpha|} D^{\alpha} u_{\text {, }}$ converge faiblement dans $L^{2}(\Omega)$, pour tout $\alpha$ fixé, vers une fonction $\psi_{\alpha}$ dépendant de $\alpha$. Mais alors, puisque $u_{y}$ converge faiblement vers une fonction $u$ au sens de $L^{2}(\mathbf{Q})$, $p^{|\alpha|} D^{\alpha} u u_{v}$ converge faiblement vers $\rho^{\alpha} D^{\alpha} u$ au sens de $\mathfrak{D}^{\prime}(\Omega)$.

Si $f$ est une distribution de $\mathbf{\Xi}^{\prime}(\mathbf{Q})$ il résulte de la Proposition 5.1 que $f=\underset{\mid \alpha, \leq l}{\Sigma} D^{\alpha}\left(\rho^{|\alpha|} f_{\alpha}\right), f_{\alpha} \in L^{2}(\Omega)$ et donc

$$
\left\langle f, u_{\nu}>=\sum_{|\alpha| \leq l}^{\Sigma}<D^{\alpha}\left(\rho^{|\alpha|} f_{\alpha}\right), u_{\nu}>=\underset{|\alpha| \leq l}{\Sigma}(-1)^{|\alpha|}<f_{\alpha}, p^{|\alpha|} D^{\alpha} u_{\nu}>\right.
$$

et l'on a done

$$
\lim _{\nu \rightarrow \infty}\left\langle f, u_{\nu}\right\rangle=\sum_{|\alpha| \leq l}^{\Sigma}(-1)^{|m|}\left\langle f_{\alpha}, \rho^{\alpha} D^{\alpha} u>\right.
$$

c'est à dire $u_{y}$ converge faiblement dans $\Xi(\Omega)$; c. q.f.d.

\section{Choix de la forme $L$.}

6. 1. Notons d'abord la

Proposition 6. 1. - Pour $v \in \mathscr{F}_{A^{*}, B^{*}}$, on $a: C_{j}^{*} v \in \mathfrak{H}(\mathbf{\Gamma})$, l'application $v \rightarrow C_{j}^{*} v$ étant linéaire continue.

\section{DÉmonstration.}

Si $v \in \mathscr{F}_{A^{*}, B^{*}}$, alors, par définition, $A^{*} v$ est nulle dans un voisinage de $\Gamma$ et $B_{j}^{*} v=0$ sur $\Gamma, j=0,1, \ldots, m-1$. Du theorème de Morrey-NirenberG [16] pour le cas du problème du. Dirichlet, étendu aux autres conditions 
aux límites dans [15] (19), il résulte que $v$ est analytique dans un voisinage de $\mathrm{T}$, done $C_{j}^{*} v \in \mathfrak{H}(\Gamma)$.

Les espaces $\mathscr{F}_{A^{*}, B^{*}}$ et $\mathscr{H}(\Gamma)$ sont $d u$ type $(\mathfrak{L F})$ au sens de [8]; le théoréme du graphe fermé est applicable (cf. [8], Théorème $B$, p.17); or l'application $v \rightarrow C_{j}^{*} v$ est par exemple continue de $\widetilde{F}_{A^{*}, B^{*}}$ dans $C^{\circ}(\mathbf{T})$, done continue de $\mathfrak{F}_{A^{*}, B^{*}}$ dans $\mathfrak{H}(\Gamma)$, d'après le théorème da graphe fermé.

Corollaire 6. 1. - Si les $g_{j}, j=0,1, \ldots, m-1$, sont données dans $\mathfrak{H}^{\prime}(\Gamma)$, la forme anti-linéaire

$$
v \rightarrow L_{1}(v)=\sum_{j=0}^{m-1}<g_{j}, \overline{C_{j}^{*} v}>
$$

(les crochets désignant la dualité entre $\mathfrak{H}^{\prime}(\Gamma)$ et $\mathscr{H}(\Gamma)$ ) est continue $\operatorname{sur} \mathscr{F}_{A^{*}, B^{*}}$.

6.2. - Comme $\mathscr{F}_{A^{*}, B^{*}} \subset \Xi(\Omega)$, (définition de $\Xi(\Omega)$ au $N^{\circ} 5$ ), avec injection continue, on a:

$$
\left\{\begin{array}{l}
\text { Si f est donné dans } \Xi^{\prime}(\mathbf{Q}), \text { la forme anti-linéaire } \\
\qquad v \rightarrow L_{2}(v)=\langle f, \bar{v}\rangle \\
\text { est continue sur } \mathfrak{F}_{A^{*}, B^{*}} \text {, le crochet désignant la } \\
\text { dualité entre } \Xi^{\prime}(\Omega) \text { et } \Xi(\mathbf{\Omega}) \text {. }
\end{array}\right.
$$

Nous prenons maintenant, avec les notations de (6.1) et (6.2):

$$
L(v)=L_{1}(v)+L_{2}(v)
$$

Si l'on fait l'hypothèse:

$$
<f, \bar{v}>+\sum_{j=0}^{m-1}<g_{j}, \overline{C_{j}^{*}} v>=0 \text { pour tout } v \in N^{*}
$$

(12) Signalons que le raisonnement de Monrey-Nirenberg, dans la généıalisation donnee dans [15], n. 13, est valable dans la seule hypothèse 13. I de [15] et que celle ci peut elle mème être généralisée, comme on voit facilement, de la façon suivante (on utilise les notations de [15])

$$
\begin{aligned}
d_{0, R_{0}}^{2}\left(\varphi_{r, h} u\right) \leq c_{2}\left\{\left\|A^{\prime}\left(\varphi_{r, h} u\right)\right\|_{0, \omega R_{0}}^{2}+\Sigma\left\|L_{i}\left(\varphi_{r, h} u\right)\right\|_{i}^{2}+\frac{1}{2}, \pi_{0}+\right. \\
\\
\left.+\left\|\varphi_{r, h} u\right\|_{0, \omega R_{0}}^{2}\right\}
\end{aligned}
$$

Or cette majoration est valable dans nos hypotèses pour $A$ et les opérateurs $L_{i}=B_{i}$ (of. $[1]$ et [20]). 
alors (6.3) définit une forme anti-linéaire continue sur $F_{A^{*}, B^{*} / N^{*}}(\mathrm{si}$ $\left.v \cdot \in \mathbb{F}_{A^{*}, B^{*}} /_{N^{*}}, v \in v \cdot, L(v \cdot)=L(v)\right)$.

En combinant les remarques précédentes au Théorème 4.1 , on a le:

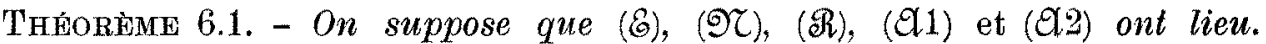
Soient $f$ donnée dans $\mathbb{\Xi}^{\prime}(\Omega), g_{j}$ données dans $\mathfrak{H}^{\prime}(\Gamma), j=0,1, \ldots, m-1$, avec (6.4). Il existe alors un élément $u$ de $\mathfrak{D}^{\prime}(\mathbf{\Omega}) / N$ et un seul tel que

$$
<u, \overline{A^{*} v}>=<f, \bar{v}>+\sum_{j=0}^{m-1}<g_{j}, \overline{C_{j}^{*} v}>
$$

pour tout $v^{*} \in F_{A^{*}, B^{*} / N^{*}}\left(v\right.$ quelconque dans $\left.v^{*}\right)$. En outre l'application $\{$, $\left.g_{n}, \ldots, g_{m-1}\right\} \rightarrow u$ est linéaire et continue du sous espace fermé de $\Xi^{\prime}(\boldsymbol{Q}) \times\left(\mathcal{H}^{\prime}(\Gamma)^{m}\right.$ formé des éléments satisfaisant à $(6.4)$ dans $\mathfrak{D}^{\prime}(\mathbf{\Omega}) / /_{N}$.

REMARQUE 6.1.

Le problème résolu dans (6.5) est d'autant plus général que $f$ est pris dans un espace «plus grand》, donc que $\bar{\Xi}(\boldsymbol{Q})$ est «plus petit». Le choix de $\Xi(\Omega)$ fait ci dessus n'est sûrement pas le choix optimum (à supposer $q^{\prime}$ ' un tel choix optimum existe) puisque $\Xi(\Omega)$ ne dépend pas de $A^{*}$ et des conditions aux limites $\ll B_{j}^{*} v=0 »$. Plus généralement on peut prendre com. me espace $\Xi(\Omega)$ un espace normal quelconque de distributions dans $\Omega$ tel que

$$
\mathbb{F}_{A^{*}, B^{*}} \subset \Xi(\Omega)
$$

avec injection continue. Le choix que nous avons fait de $\Xi(\Omega)$ simplifie les No suivants.

6.3. - Le problème qui nous reste à résoudre est maintenant l'interprétation de (6.5). Il nous faut pour cela obtenir de nouveaux théorèmes de traces; cela sera fait au $N^{\circ} 8$, les résultats préliminaires pour cela étant donnés au No suivant.

\section{7. - Lemmes préliminaires pour la définition des traces.}

Dans l'interprétation de $(b .5)$ on sera amené à considérer l'espace $D_{\Delta}$ des $u \in \mathfrak{D}^{\prime}(\mathbf{\Omega})$ telles que $A u \in \mathbb{\Xi}^{\prime}(\mathbf{\Omega})$. On munit cet espace de la topologie localement convexe la moins fine rendant continues les applications $u \rightarrow u$ et $u \rightarrow A u$ de $D_{A}$ dans $\mathscr{D}^{\prime}(\mathbf{\Omega})$ et $\boldsymbol{\Xi}^{\prime}(\boldsymbol{\Omega})$ respectivement. On a le: 


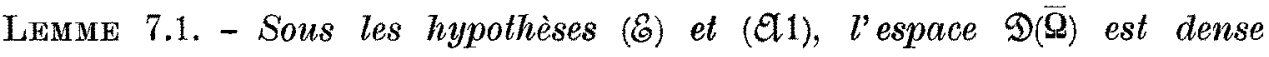
dans $D_{A}$.

\section{DÉMONSTRATION.}

Soit $u \rightarrow M(u)$ une forme anti-linéaire continue sur $D_{A}$. Alors $M(u)$ est de la forme (noter que $\boldsymbol{\Xi}(\boldsymbol{\Omega})$ est réflexif)

$$
M(u)=\langle f, \bar{u}\rangle+\langle g, \overline{A u}\rangle, f \in \mathfrak{D}(\Omega), g \in \Xi(\Omega)
$$

Supposons que $M(\varphi)=0$ pour tout $\varphi \in \mathfrak{D}(\overline{\mathbf{Q}})$; nous voulons montrer que dans ces conditions $M(u)=0$ pour tout $u \in D_{\boldsymbol{A}}$.

Or, si $p \in \mathscr{D}(\overline{\mathbf{\Omega}})$, il existe $\Phi \in \mathscr{D}\left(R^{n}\right)$, telle que $\Phi=\varphi$ sur $Q$. Soient par ailleurs $\tilde{f}$ et $\tilde{g}$ les prolongements de $f$ et $g \grave{a} R^{n}$ par 0 hors de $Q$, et $\mathfrak{Q}$ le prolongement de $A$, à coefficients analytiques dans $R^{n}$, $\mathfrak{G}$ vérifiant (q) dans un voisinage $\mathcal{O}$ de $\overline{\boldsymbol{\Omega}}$.

Alors

$$
M(\varphi)=<\tilde{f}, \bar{\Phi}>+<\tilde{g}, \overline{\mathfrak{g} \Phi}>=0
$$

done

$$
<\mathfrak{A}^{*} \tilde{g}+\tilde{f}, \bar{\Phi}>=0 \quad(\mathfrak{Q} * \text { adjoint formel de } \mathfrak{a})
$$

et ceci pour tout $\Phi \in \mathscr{D}\left(R^{n}\right)$; done

$$
\mathfrak{A}^{*} \tilde{g}=-\tilde{f} \quad \text { dans } R^{n}
$$

Alors, d'après l'hypoellipticité de $\mathfrak{Q}^{*}$ dans $\mathcal{O}, \tilde{g}$ est indéfiniment diffé. rentiable dans $\Theta$; et comme $\tilde{f}$ est analytique (puisque nulle!) dans $\mathrm{C} k, k$ compact de $Q, \tilde{g}$ est également, d'après (7.2), analytique dans $\mathcal{O} \cap \mathrm{G} k$; comme $\tilde{g}$ est nulle dans $\mathcal{O}-\mathbf{\Omega}$, on $a: \tilde{g}=0$ dans $\mathbb{C} k$, donc $g \in \mathfrak{D}(\boldsymbol{Q})$. En outre, par restriction de (7.2) $\dot{\mathrm{d}} \boldsymbol{\Omega}$ :

$$
A^{*} g=-f \quad \text { dans } \Omega .
$$

Mais alors, dans $(7.1),\langle g, \overline{A u}\rangle=\left\langle A^{*} g, \bar{u}\right\rangle \operatorname{car} g \in \mathfrak{D}(\mathbf{\Omega})$, et

$$
M(u)=\left\langle f+A^{*} g, \bar{u}\right\rangle\left(\text { dualité entre } \mathfrak{D}(\mathbf{\Omega}) \text { et } \mathfrak{D}^{\prime}(\mathbf{\Omega})\right)
$$

d'après $(7.3)$, on a donc $M(u)=0$, ce qui achève la démonstration du Lemme. 


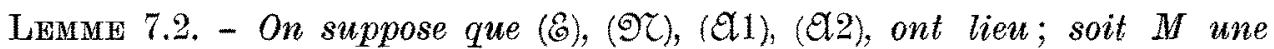
constante $>0$ fixée; on considère l' espace $\mathscr{H}_{M, 0}(\Gamma)$ introduit au $\mathrm{N}^{\circ} 1$, point 1.1 . Soient $\varphi_{j}, j=0,1, \ldots, m-1$, données dans $\mathfrak{H}_{M, 0}(\mathbf{I})$. Il existe alors $v \in \mathfrak{F}_{A^{*}, B^{*}}$, tel que

$$
C_{j}^{*} v=\varphi_{j}, \quad j=0, \ldots, m-1 .
$$

On peut choisir $v$ de façon que l'application

$$
\vec{\varphi}=\left\{\varphi_{0}, \ldots \cdot \varphi_{M-1}\right\} \rightarrow v=v \overrightarrow{(\varphi)}
$$

soit linéaire et continue de $\left(\mathcal{H}_{M, 0}(\Gamma)\right)^{m}$ dans $\mathscr{F}_{A^{*}} . B^{*}$. (La fonction $v$ dépend aussi de $M$, mais $M$ est fixé dans cet énoncé; on aurait un énoncé analogne avec $\mathscr{H}_{\boldsymbol{M}, 2}(\Gamma)$ au lieu de $\left.\mathscr{H}_{\boldsymbol{M}, 0}(\Gamma)\right)$.

\section{DÉmonstration.}

1) Il faut construire $v$ telle $q u$ ue $v \in \mathscr{D}(\overline{\mathbf{\Omega}})$, avec

$$
\left\{\begin{array}{lll}
B_{j}^{*} v=0 & \text { sur } \Gamma, & j=0, \ldots, m-1 \\
C_{j}^{*} v=\varphi_{j} & \text { sur } \Gamma, & j=0, \ldots, m-1
\end{array}\right.
$$

et $A^{*} v \in \mathfrak{D}(\boldsymbol{Q})$.

D'après un Lemme de Aronszajn-Milgram [2] (le système $\left\{B_{j}^{*}, C_{j}^{*}\right\}$ étant un système de DiRICHLET), les conditions (7.5) sont équivalentes aux conditions suivantes:

$$
\gamma_{j} v=\psi_{j} \text { sur } \mathrm{T}, \quad j=0,1, \ldots, 2 m-1
$$

où

$$
\gamma_{j}=\text { dérivée normale d'ordre } j
$$

et où les $\psi_{j}$ sont des fonctions convenables; il existe un nombre $N$ tel que si $\varphi_{j} \in \mathscr{H}_{M, 0}(\Gamma)$ alors $\psi_{j} \in \mathscr{H}_{N, 0}(\Gamma)$.

2) Considérons maintenant le problème de Cauchy:

$$
\begin{cases}A^{*} u=0 & \text { dans un voisinage de } \mathrm{T}, \\ \gamma_{j} u=\psi_{j} & \text { sur } \Gamma, j=0,1, \ldots, 2 m-1 .\end{cases}
$$


J. L. Lions - E. Magenes: Problèmes aux limites non homogènes (VII) 219

D'après le théoreme de Cauchy-Kovalewska, et $\Gamma$ étant compacte (et tous les coefficients analytiques) le problème (7.7) admet une solution unique dans un voisinage fixé de $\Gamma$ (ce voisinage dépendant de $N$, i. e. de $M$, mais non du choix des $\varphi_{j}$ pourvu que $\left.\varphi_{j} \in \mathscr{H}_{M, 0}(\Gamma)\right)\left({ }^{13}\right)$. Done si l'on désigne par $l_{\rho}$ l'ensemble des points de $Q$ tels $q u e d(x, \Gamma)<\rho, \rho$ assez petit, on voit qu'il existe $\rho(M)$ tel que (7.7) admette une solution unique dans $I_{\rho(M)}$.

Soit alors $\alpha$ une fonction de $\mathscr{D}(\overline{\mathrm{Q}})$, telle $q$ ue $\alpha=0$ dans $\bar{\Omega}-I_{p}(M)$ et $\alpha(x)=1$ dans $I_{\rho\langle M\rangle_{2}}$; une telle $\alpha$ existe évidemment.

Posons enfin :

$$
v=\left\{\begin{array}{rll}
\alpha u & \text { dans } & I_{\rho(M)}, u \text { solution de }(7.7) \\
0 & \text { dans } & \Omega-I_{\rho(M)} .
\end{array}\right.
$$

Alors (on a fait ce qu'il fallait pour ça!), (7.6) a lieu, donc (7.5) a lieu. Enfin $v \in \mathfrak{D}(\bar{\Omega})$ et $A^{*} v=x A^{*} u=0$ dans $I_{P(M) / 2}$ done $A^{*} v \in \mathfrak{D}(\Omega)$.

On a done défini une application linéaire de $\left(\mathfrak{H}_{M, 0}(\Gamma)\right)^{m}$ dans $\mathscr{F}_{A^{*}, B^{*}}$ dont il reste seulement à montrer la continuité.

3) Mais les espaces $\left(\mathfrak{d}_{M, 0}(\boldsymbol{\Gamma})\right)^{m}$ et $\mathscr{F}_{A^{*}, B^{*}}$ étant en particulier des espa-

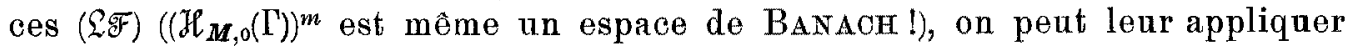
le théorème du graphe fermé - Mais, du théorème de Cadchy-Kovalewska résulte la continuité de l'application:

$$
\left\{\psi_{j} \mid j=0, \ldots, 2 m-1\right\} \rightarrow u=\text { solution de }(7.7)
$$

de $\left(\mathscr{H}_{N, 0}(\Gamma)\right)^{2 m}$ dans $C^{\circ}\left(\overline{\boldsymbol{I}}_{\varphi(\boldsymbol{M})}\right)$ (espace des fonctions continues dans $\left.\overline{\boldsymbol{I}}_{\boldsymbol{\alpha}(\boldsymbol{M})}\right)$, d'où aussitòt le résultat.

(13) Il est, peut etre, atile de signaler de quelle façon on applique le thérème de CaUChy - Kowalewska: les fonctions de JiM, $0(\Gamma)$ vérifient, après application des homéo. morphismes $\theta_{i}$ (cf. n. 1, Prop. 1.1), les conditions habitalles portant sur toutes les derivées,

$$
\left|\frac{\partial^{k} f(y)}{\partial y_{1}^{k_{1}} \ldots \partial y_{n-1}^{k_{n}-1}}\right| \leq k_{1} ! \ldots k_{n} ! \tilde{L} \tilde{M}^{k}, y \in S^{n-1},
$$

avee une valeur de la constante $\tilde{M}$ dépendant de $M$ (ceci d'après le thèoréme de KotaKENarasinhau [9]); on peut appliqner alors le theorème de CaUchy-Kovalewsira dans la situation classique, après avoir utilise un système de "cartes locales,, d'un voisinage de I dans $R^{n}$, lié de façon évidente aux systèmes de coordonnées locales sur $\mathbb{R}$. 


\section{8. - Un théorème de traces.}

Nous allons dans ce $\mathrm{N}^{\circ}$ démontrer le théorème de traces suivant:

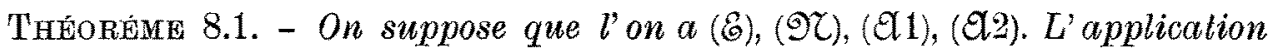

$$
u \rightarrow B u=\left\{B_{0} u, B_{1} u, \ldots, B_{M-1} u\right\}
$$

de $\mathscr{D}(\overline{\mathbf{\Omega}})$ dans $(\mathcal{D}(\mathrm{T}))^{m}(\mathcal{D}(\mathrm{\Gamma})$ est l'espace des fonctions indéfiniment différentiables sur $\Gamma)$ se prolonge par continuité en une applicalion linéaire continue, encore notée $u \rightarrow B u$, de $D_{A}$ dans $\left(\mathfrak{P}^{\prime}(\mathrm{\Gamma})\right)^{m}$.

En outre, pour $u \in D_{\mathcal{A}}$ et $v \in \mathscr{F}_{\mathcal{A}^{*}, B^{*}}$ on a la formule de Green:

$$
<A u, \ddot{v}>-<u, \overline{A^{*} v}>=-\sum_{j=0}^{m-1}<B_{j} u, \overline{C_{j}^{*} u}>
$$

Coù le premier crochet du premier membre désigne la dualité entre $\Xi^{\prime}(\Omega)$ et $\Xi(\Omega)$, car $v \in \mathscr{F}_{A^{*}, B^{*}} \subset \Xi(\Omega)$; où le denxième crochet désigne la dualité entre $\mathfrak{D}^{\prime}(\mathbf{\Omega})$ et $\mathfrak{D}(\Omega)$, et où les orochets du $2^{\text {òme }}$ membre désignent la dualité entre $\mathscr{H}^{\prime}(\mathbf{T})$ ef $\mathcal{H}(\Gamma)$ ).

\section{DÉMONSTRATION.}

1) Soit $u$ donné dans $D_{A}$. Soit $\vec{\varphi}=\left\{\varphi_{0}, \ldots, \varphi_{m-1}\right\}$ donné dans $\left(\mathcal{H}(\Gamma)^{m n}\right.$. Alors $\vec{\varphi} \in\left(\mathcal{H}_{M, 0}(\Gamma)\right)^{m}$ pour un $M$ convenable; nous choisissons $v=v(\vec{\varphi})$ comme au Lemme 7.2, et nous introduisons

$$
Y(v \overrightarrow{\varphi(\varphi)})=<u, \overline{A^{*} v(\vec{\varphi})}>-<A u, \overrightarrow{v(\vec{\varphi})} \text { 之, }
$$

le premier crochet désignant la dualité entre $\mathscr{D}^{\prime}(\Omega)$ et $\mathscr{D}(\boldsymbol{\Omega})$, le deuxième entre $\boldsymbol{\Xi}^{\prime}(\mathbf{\Omega})$ et $\boldsymbol{\Xi}(\boldsymbol{\Omega})$.

Vérifions que $Y(\vec{v}(\varphi)$ ne dépend que de $\vec{\varphi}$. Soit en effet $w$ une autre fonction de $\mathscr{F}_{A^{*}, B^{*}}$ satisfaisant à $C^{*}{ }_{j} w=\varphi_{j}, 0 \leq j \leq m-1$; alors $\chi=v-w$ satisfait à $B_{j}^{*} \chi=0, C_{j}^{*} \chi=0,0 \leq i \leq m-1$, donc, d'après le Lemme de Aronszajn - Milgram, à $\gamma_{j} \chi=0,0 \leq j \leq 2 m-1$, et comme $A^{*} \chi \in \mathscr{D}(\mathbf{Q})$, il résulte, d'après l'unicité du problème de CAUCHY (7.7), que $\chi \in \mathscr{D}: 9$ ). Mais alors

$$
<u, \overline{A^{*} v}-\overline{A^{*} v}>=<A u, \bar{\chi}>\text {, done } Y(v)=Y(v)
$$

On peut donc poser:

$$
Y(v(\vec{\varphi}))=Z \overrightarrow{(\varphi)} .
$$


2) La forme $\vec{p} \rightarrow Z(\vec{p})$ est linéaire sur $(\mathfrak{H}(\Gamma))^{m}$; vérifions par exemple que $Z\left(\vec{\varphi}_{1}+\vec{\varphi}_{2}\right)=\left\langle\left(\overrightarrow{\varphi_{1}}\right)+Z\left(\overrightarrow{\varphi_{2}}\right) ;\right.$ l'on peat trouver $M$ tel que $\vec{\varphi}_{1}, \vec{\varphi}_{2} \in\left(H_{M, 0}(\Gamma)\right)^{n}$, et on utilise alors (8.3) pour la délinition de $Y($ ou $Z)$; alors la linéarité résulte de la linéarité de l'application (7.4).

Montrons maintenant que $\vec{\varphi} \rightarrow Z\left(\overrightarrow{p)}\right.$ est continue sur $(\mathscr{H}(\mathbf{\Gamma}))^{m}$. Il suffit (cf. [23]) de vérifier que $\vec{\varphi} \rightarrow Z\left(\overrightarrow{p)}\right.$ est continue sur $\left(\mathscr{H}_{M, 0}(\Gamma)\right)^{m}, M$ fixé. On définit alors $h(\varphi)=Y(v(\varphi))$ par $(8.3)$ et la continuité résulte alors du Lemme 7.2.

3) Par conséquent, on a :

$$
Z\left(\overrightarrow{\varphi)}=\sum_{j=c}^{m-1}<\tau_{j} u, \bar{\varphi}_{j}>, \quad \tau_{j} u \in \mathscr{H}^{\prime}(\Gamma)\right.
$$

et l'application

$$
u \rightarrow \tau u=\left\{\tau_{0} u, \ldots, \tau_{m-1} u\right\}
$$

est linéaire de $D_{A}$ dans $\left(\mathfrak{H}^{\prime}(\mathrm{\Gamma})\right)^{m}$.

Montrons maintenant la continuité de l'application (8.6). Il suffit montrer que, étant donné un borné $\mathcal{B}$ de $\left(\mathcal{H}(\Gamma)^{m}\right.$; il existe un voisinage de 0 dans $D_{A}$, soit 2), tel que

$$
\left.|<\tau u, \overline{\vec{\varphi}}>|=\sum_{j=0}^{m-1}<\tau_{j} u, \bar{\varphi}_{j}>1 \leq 1 \quad \text { pour tout } u \in \mathcal{Q}\right), \vec{\varphi} \in B
$$

Mais $\mathfrak{B}$ est nécessairement un borné de $\left(\mathcal{H}_{M, 0}(\Gamma)\right)^{m}$ pour un $M$ convenable; choisissons $v(\overrightarrow{\varphi)}$ pour cet $M$ comme au Lemme 7.2; alors

$$
<\tau u, \overline{\vec{\varphi}}\rangle=\left\langle u, \overline{A^{*} v(\vec{\varphi})}\right\rangle-\langle A u, \overline{v(\vec{\varphi})}\rangle
$$

et $v(\vec{\varphi})$ demeure dans un borné de $\vec{F}_{A^{*}, B^{*}}$, donc de ${\overrightarrow{y_{Y}}}_{\mathbf{A}^{*}, B^{*}}^{\alpha} \alpha$ fixé, et donc, en particulier, $\overrightarrow{v(\varphi)}$ demenre dans un borne $\mathfrak{B}_{i}$ de $\mathscr{D}(\overline{\mathbf{\Omega}})$ et $A^{*} \overrightarrow{v(\varphi)}$ dans un borne $\mathfrak{B}_{z}$ de $\mathfrak{D}(\boldsymbol{Q})$; notons que $\mathfrak{B}_{1}$ est borné dans $\Xi(\Omega) ; X^{\circ}$ désignant le polaire de $X$, on peut alors prendre

$$
\mathscr{D})=\left\lfloor\mid u \in \frac{1}{2} \mathfrak{B}_{2}^{0}, A u \in \frac{1}{2} \mathfrak{B}_{1}^{0} \subset \mathbb{\Xi}^{\prime}(\mathbf{Q})^{\prime}\right.
$$

Le résultat suit.

4) Prenant maintenant $u \in \mathfrak{D}(\overline{\mathbf{Q}})$ et atilisant la formule de Grewn, on voit tout de suite que $\tau u=B u$, ce qui démontre la première partie du Théorème.

En outre on a obtenn

$$
<u, \overline{A^{*} v(\vec{\varphi})}>-<A u, \overrightarrow{v(\vec{\varphi})}>=\sum_{j=0}^{m-1}<B_{j} u, \overline{\varphi_{j}}>
$$


mais $\varphi_{j}=C_{j}^{*} v(\vec{\varphi})$ et posant $v(\vec{\varphi})=v$, on obtient donc (8.2), ce qui achève la démonstration du Théorème.

\section{Problèmes aux limites non homogènes.}

Revenons maintenant à l'équation (6.5). Soit $u \in \in \mathscr{D}^{\prime}(\boldsymbol{\Omega}) /_{N}$ satisfaisant à $(5.5)$; prenons $v \in \mathfrak{D}(\Omega)$ et soit $v$ la classe définie par $v$ dans $\mathscr{F}_{A^{*}}, B^{*} / N^{*}$. Alors (6.5) donne

$$
<u, \overline{A^{*} v}>=<f, \bar{v}>, u \in u^{\prime},
$$

donc

$$
<A u-f, \bar{v}>=0
$$

done

$$
\text { A } u=f
$$

Par conséquent, $u$ étant quelconque dans $u^{*}, u \in \mathfrak{D}^{\prime}(\mathbf{\Omega})$ et $A u=f \in \mathbb{\Xi}^{\prime}(\boldsymbol{\Omega})$; done $u \in D_{A}$. Mais alors la formule de Green (8.2) est valable. Donc

$$
\left.<A u, \bar{v}\rangle=\langle f, \bar{v}\rangle=\left\langle u, \overline{A^{*} v}\right\rangle-\sum_{j=0}^{m-1}<B_{j} u, C_{i}^{*} \bar{v}\right\rangle
$$

de sorte que, en comparant avec $(6.5)$, on a:

$$
\sum_{j=0}^{m-1}<g_{j}-B_{j} u, \overline{C_{i}^{*} v}>=0
$$

pour tout $v \in v, v^{*} \in F_{A^{*}}, E^{*} / N^{*}$. Mais d'après le Lemme $7.2,(9.2)$ équivaut à

$$
\sum_{j=0}^{m-1}<g_{j}-B_{j} u, \bar{\varphi}_{j}>=0 \quad \text { pour tout } \varphi_{j} \in \mathcal{H}(\mathbf{\Gamma})
$$

done

$$
B_{j} u=g_{1}
$$

Done, si l'on désigne par $\left\{\overline{\boldsymbol{\Xi}}^{\prime}(\mathbf{\Omega}) \times\left(\mathcal{H}^{\prime}(\Gamma)\right)^{m} ; N^{*}, C N^{*}\right\}$ l'espace des $\left\{f, g_{0}, \ldots\right.$, $\left.g_{m-1}\right\}, f \in \Xi^{\prime}(\boldsymbol{\Omega}), g_{j} \in \mathfrak{H}^{\prime}(\boldsymbol{\Gamma})$. tels que (6.4) ait lieu, on a le

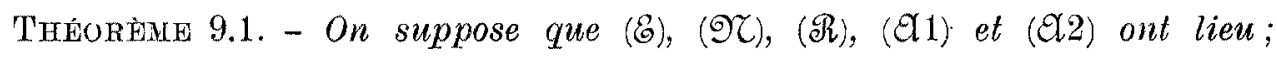


J. L. Lrons - Q. Marmass: Problimes anx limites non homogrnes (VII) 223

\section{l'application}

$$
u \cdot \rightarrow\left\{A u, B_{0} u, \ldots, B_{m-1} u\right\}
$$

est un isomorphisme de $D_{A^{\prime} /}$ sur $\left\{\Xi^{\prime}(\Omega) \times\left(\mathfrak{H}^{\prime}(\mathrm{T})^{n n} ; N^{*}, C N^{*}\right\}\right.$.

Autrement dit, pour $f \in \Xi^{\prime}(\boldsymbol{Q}), g_{1} \in \mathcal{H}^{\prime}(\boldsymbol{\Gamma})$, avec $(6 \cdot 4)$, il existe un elément $u$ de $D_{A}$, unique modulo $N$, tel que l'on ait (9.1) et (9.2); en outre $u$. dépend continûment de $f$ et $g_{j}$. Pour le problème (2.2) on a done encora le théorème de l'alternative sous sa forme habituelle, même dans ces espaces très généraux.

\section{BIBLIOGRAPHIE}

[1] S. AgMon - A. Douglis - L. Nirenzerg, Estimates near the boundary for solutions of elliptic partial differential equations satisfying general boundary conditions, "Comm. pure applied. math. ", 12 (1959), 623.727.

[2] N. Aronszajn - A. N. Mitgram, Differential operators on Riemannian manifolds, "Rend. Circ. Mat. Palermo », 2 (1952), 1.61.

[3] F. E. BRowDER, Estimates and existence theorems for elliptic boundary value problems, "Proc. Nat. Acad. Sc. U. S. A.», 45 (1959), 365.372.

[4] G. Cimmino, Su alcuni esempi rotevoli di dualità fra spazi lineari topologici ", à paraitre aux Rend. Sem. Mat. Fis. Milano, (1962).

[5] G. De Rham, Variétes différentiables, "Hermann, Paris, " 1955.

[6] J. Dieudonné - L. Sohwartz, La dualité dans les espaces (F্F) et (LF) , Annales Institut Fourier ", t. 1 (1950), 61-101.

17] I. M. GELFAND - G. F. Sonnov, Fonctions généralisées (distributions), (en russe), «vol. 1,..., 17, Moscous, 1958 .

[8] A. Grothendrek, Produits tensoriels topologiques et espaces nucléaires, "Memoirs of the Amer. Math. Soc», No 16, 1955.

[9] T. KotakE - M. S. NarasinaAm, Fractional poners af a linear elliptic operator, \& Bull. Soc. Math. France ", t. 90 (1962) 449.471

[10] G. KöTHE, Die Stufenraume, eine einfache Klasse linearer vollkommener Räumen, "Math. Zeitsch. », t. 51 ( 948), $317 \cdot 345$.

[11] G. Köтне, Die Randverteillungen analytischer funtionen, "Math. Zeitsch.s, t. 57 $(1957 \cdot 58), 13-33$.

[12] G. Körue, Topologische lineare Räume, «Springer, Berlin », 1960.

[13] J. L. LIONS - F. MAGENES, Froblèmes aux limites non homogènes. (I)...(VI); (I), (IIT); (IV), (V). "Annali Senola Norm. Sup. Pisa », t. XIV (1:60), 259-308; t. XV (1961), 39.101, 311.326; t. XVI (1962), 1.44; (II), Annales Instifut Fourier, t. 11 (1961), 137.178; IVI) Journal d'Analyse Mathématique, XI (1963) $16 \%$ \%ั188.

[14] J. L. LIONs - E. Magenes, Remarques sur les problèmes aux limites linéaires elliptiques, "Rend. Acc. Lincei ", t. XXXII (1962), 873-883.

[15] E. Magenes - G. StampaCchia. I problemi al contorno per le equazioni differenziali di tipo ellittico, "Annali Scuola Norm. Sup. Pisa", vol. XII, (1958), 247.358.

[16] C. B. Morrey - L. Nirenberg, On the analyticity of the solutions of linear elliptic systems of partial differential equations, «Comm. Pure Applied Math.» 10 (1957), 271.290.

[17] E. Nelson, Anvlytic vectors, "Annals of Math. ", t. 70 (1959), 572.615. 
[18] C. Roumieu, Sur quelques extensions de la notion de distribution, "Annales Scient. Ec. Norm. Sup. s, t. 77 (1960), 47.121.

[19] C. Roumru, Ultra-distribulions definies sur $R^{n}$ et sur certaines classes de variétés différentiables, Journal d'Analyse Math. vol. X ", (1962, 63), 153.192.

[20] M. Schechter, General boundary value problems for elliptic partial differential equations, «Comm. pure applied math. », 12 (1959), 4:57.486.

[21] L. Schwartz, Thérie des distributions, I et II. "Hermann Paris s, 1950.1051.

[22] H. G. TILLMAN, Randverteilungen analytischer Funktionen und distributionen, "Math. Zeitsch », 59 (1953), 61.83.

[23] N. BourbaxI, Espaces vectoriels topolvgiques. "chap. I et II, Hermann, Paris ^, 1933.

[24] I. SeBastiao e Silva, Su certe classi di spazi localmente convessi importanti per le applicazioni, "Rend. di Mat. », s. IV, XIV, (190̄5), $\$ 88.410$.

\section{INDEX DES NOTATIONS PRINCIPALES}

Si $\Delta=$ opérateur de Laplace Beltrami sur $\Gamma$, on définit

$$
\mathfrak{H}_{M}, \circ(\Gamma)=|f| \sup _{k} \frac{1}{(2 k) ! M^{k}}\left\|\Delta^{k} f\right\| C^{0}(\Gamma)=\|f\|_{\mathfrak{H}_{M, o}(\Gamma)}<\infty \mid
$$

$\mathfrak{Z}(\Gamma)=$ limite inductive $\operatorname{des} \mathfrak{H}_{\boldsymbol{M}}, \mathrm{o}(\mathrm{N})$ :

$\mathfrak{H}^{\prime}(\Gamma)=$ dual fort $\mathrm{de} \mathscr{H}(\Gamma)$ (fonctionnelles analytiques sur $\Gamma$ ).

$\mathscr{D}(\Omega)($ resp. $\mathscr{D}(\bar{\Omega}))=$ fonetions indéfiniment différentiables dans $Q$ (resp. $\bar{\Omega}$ ) à support compact dans $\mathbf{Q}$ (resp. quelconque dans $\overline{\mathbf{\Omega}}$ ), topologie de limite inductive de L. SCHwarTz (resp. d'espace de Fréchet).

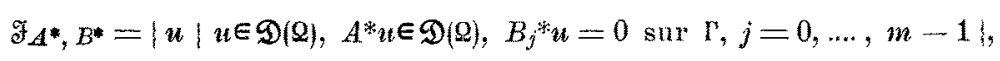

topologie de limite inductive des $\mathscr{F}_{A^{*}}^{*}, B^{*}$ (of. p. 11).

$\Xi(\Omega)=|u| \rho|\alpha| D^{\alpha} u \in L^{2}(\Omega)$ pour tout $\left.\alpha\right\}$ topologie naturelle d'espace de Fréchet, $\rho=$ fonction équivalente à le distance au bord de $\Omega$ (cf. p. 12); $\Xi^{\prime}(\Omega)=$ dual fort de $\Xi(\Omega)$.

$D_{A}=\left\{u \mid u \in \mathfrak{D}^{\prime}(\Omega), A u \in \mathbb{E}^{\prime}(\Omega)\right\}$, topologie la moins fine rendant continues les applica. tions $u \rightarrow u, u \rightarrow A u$. de $D_{A} \rightarrow \mathfrak{D}(\Omega)$ et $D_{A} \rightarrow \mathbb{Z}^{\prime}(\Omega)$. 